\title{
Startup Qilimanjaro-towards a European full-stack coherent quantum annealer platform
}

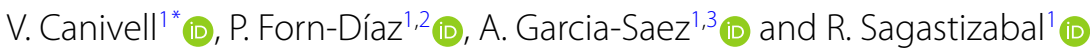

"Correspondence:

victor.canivell@qilimanjaro.tech

'Qilimanjaro Quantum Tech S.L., Barcelona, Passeig de Gràcia 58, 08007 Barcelona, Spain

Full list of author information is

available at the end of the article

\section{Springer}

\begin{abstract}
Qilimanjaro Quantum Tech is the full-stack quantum spin-off of three research institutions, the Barcelona Supercomputing Center (BSC), the Institute for High Energy Physics (IFAE) and the University of Barcelona (UB). The company addresses the emerging quantum readiness demand from industry and academia, by providing both algorithmic development services as well as access to a new coherent quantum annealer platform, a special purpose quantum computer. Qilimanjaro is a member of the AVaQus European Commission's H2020 FET-Open consortium for coherent quantum annealing development, which is led by one of Qilimanjaro's founders at IFAE. A special feature of Qilimanjaro are its funding sources being exclusively international client contracts.
\end{abstract}

\section{Introduction}

Quantum computing has recently emerged from the academic realm into the business arena. As every region in the world ${ }^{1}$ starts to develop their participation in this market, Europe is not the exception. ${ }^{2}$ In parallel to countries such as the United States ${ }^{3}$ and China, ${ }^{4}$ Europe has also committed substantial funds to aid the development of a quantum industry, and several entrepreneur teams have risen to the challenge with ambitious plans, typically leveraging the expertise of its academic centers of excellence. The goal is to successfully map leadership in research to leadership in business, both as providers of competitive and successful technology as well as of advanced users in industry.

Qilimanjaro Quantum Tech (Qilimanjaro) ${ }^{5}$ is an interesting case study for an innovative European deep-tech startup. Spinning out of three academic centres in mid 2019, Qilimanjaro was able to kick start operations with the immediate signature of international client contracts. Simultaneously, one of its founders was awarded in 2020 the direction of

\footnotetext{
${ }^{1}$ http://www.qureca.com/overview-on-quantum-initiatives-worldwide/

${ }^{2} \mathrm{http}: / / \mathrm{qt} . \mathrm{eu}$

${ }^{3}$ http://www.quantum.gov

${ }^{4}$ http://www.globaltimes.cn/content/1203759.shtml

${ }^{5}$ http://www.qilimanjaro.tech
}

(c) The Author(s) 2021. This article is licensed under a Creative Commons Attribution 4.0 International License, which permits use, sharing, adaptation, distribution and reproduction in any medium or format, as long as you give appropriate credit to the original author(s) and the source, provide a link to the Creative Commons licence, and indicate if changes were made. The images or other third party material in this article are included in the article's Creative Commons licence, unless indicated otherwise in a credit line to the material. If material is not included in the article's Creative Commons licence and your intended use is not permitted by statutory regulation or exceeds the permitted use, you will need to obtain permission directly from the copyright holder. To view a copy of this licence, visit http://creativecommons.org/licenses/by/4.0/. 
a FET-Open research project, ${ }^{6}$ whose outcome is planned to be scaled up by Qilimanjaro for market launch. Driving these three initiatives at the same time (several spin-off agreements, several client contracts and a European research project) is challenging enough for a young startup, but it certainly provides for a promising start.

The scientific core of Qilimanjaro is the brainchild of three experienced quantum physicists $^{7,8,9}$ that share a common vision to fast-track development of a European full-stack quantum platform: to effectively provide quantum advantage to certain real-world complex problems, within a time frame shorter than that estimated for error-corrected universal quantum systems. Their areas of expertise are complementary, covering knowledge on both the experimental superconducting qubit technology and the theoretical quantum and classical algorithmic fields. The scientific team is tightly knit since its members have worked and published together in the past. This scientific core team was complemented by a fourth quantum physicist turned senior IT executive ${ }^{10}$ with both multi-national and startup experience, and by another seasoned entrepreneur and M \& A executive. ${ }^{11}$ The initial objective of the founders was to study the opportunity of developing and launching a European quantum computing technology startup. This is what they did, founding the company in spring 2019 , as a result of customer demands, of the opportunity of leveraging key infrastructures and of a closely related European funded project.

Qilimanjaro is so far one of the few full-stack quantum startups that internally develops both its quantum hardware as well as its quantum software. This combination represents a very important advantage in the present initial era of the quantum industry: it can naturally tailor its software and hardware for very specific tasks, implementing the so-called co-design approach [6]. Full-stack companies are uniquely positioned to deliver this type of technological advantage.

This article is organized as follows: Sect. 2 introduces the coherent quantum annealing technology developed at Qilimanjaro, Sect. 3 presents Qibo, Qilimanjaro's software stack, while Sect. 4 describes the present and future business models developed at the company. The article closes with several concluding remarks.

\section{Full-stack coherent quantum annealing technology}

Quantum annealing is an alternative quantum computing paradigm with respect to digital quantum computing, which nowadays is the most widespread approach in the quantum computing industry as well as in academia. Quantum annealing is often considered an analog way of processing quantum information by implementation of the so-called adiabatic quantum computing algorithm.

\footnotetext{
${ }^{6}$ http://www.avaqus.eu

${ }^{7}$ Dr. J. I. Latorre-Co-Founder and Chief Science Officer \& Director of the Center for Quantum Technologies, Singapore (https://www.quantumlah.org/people/director.php) and of the Quantum Group at the Technology Innovation Institute, Abu Dhabi (https://www.tii.ae/team/prof-jose-ignacio-latorre), on leave as Professor of Theoretical Physics at the University of Barcelona.

${ }^{8}$ Dr. Pol Forn-Díaz-Co-Founder and Chief Hardware Architect \& Quantum Computing Technology Group leader at IFAE (https://qct.ifae.es)

${ }^{9}$ Dr. Artur García Sáez-Co-Founder and Chief Software Architect \& Quantum Group leader at BSC (https://www.bsc.es/garcia-saez-artur).

${ }^{10}$ Dr. Victor Canivell, Co-Founder and Chief Business Officer (https://es.linkedin.com/in/victorcanivell).

${ }^{11}$ Jordi Blasco, Co-Founder and Chief Finance Officer (https://es.linkedin.com/in/jordiblasco).
} 
Presently, the limelight for quantum computing research and development is directed towards advancing short-term road maps for various kinds of gate-based NISQ ${ }^{12}$ architectures, pointing towards longer-term, future error-correcting and fault-tolerant platforms with several qubit technologies. However, there is also significant interest in quantum annealing, especially in the US and Japan. In the US, IARPA's Quantum Enhanced Optimization (QEO) program started in June 2017 [12] and was superseded by DARPA's Quantum Annealing Feasibility Study (QAFS) program in Feb. 2020; in Japan, where initial pioneering of quantum computing adiabatic and annealing architectures took place, there is an active development pursuit at both research and commercial organizations. ${ }^{13}$ The Qilimanjaro team has decided to drive a European initiative in this area, complemented through its participation in the FET Open-funded AVaQus project, introduced at the end of this section. This is the technology window of opportunity that Qilimanjaro is seeking.

Quantum annealers have important advantages in the present NISQ era of quantum computation where quantum error correction is not yet available, hindering the faithful implementation of quantum algorithms using digital quantum gates. By contrast, quantum annealers solve optimization problems of great societal utility without the need for quantum error correction. Notable examples include scheduling, traffic flow optimization, machine learning and quantum chemistry. Quantum annealing does not operate using quantum gates, therefore errors usually associated to the fidelity of gate implementation are not relevant. In short, quantum annealers have a great short-term transformative potential, being one of the main reasons Qilimanjaro is adopting them as its basic quantum hardware platform. Despite the fact that quantum annealers may only address certain types of problems, there are routes to upgrade them to universal adiabatic quantum processors in the longer term [1].

The quantum annealing market has existed for over a decade, with current quantum annealers consisting of superconducting devices containing a few-thousand qubits [3]. Significant engineering milestones have been achieved, but demonstration of quantum advantage by leveraging true quantum effects has proven quite challenging. One of the main causes in existing annealers seems to point to the computations lasting longer than the observed quantum coherence of the qubits themselves. The circuit designs in these annealers can only implement problems which are efficiently solvable by conventional computers. As a consequence, no real quantum speedup has been detected so far. These systems are sometimes referred to as incoherent annealers. Despite their limitations, this early generation of devices have generated significant commercial interests, and many software applications have been developed to provide certain business advantages.

Qilimanjaro's technology departs from existing quantum annealers by introducing an important key ingredient: qubit coherence, which impacts the way in which the annealer processes quantum information. Therefore, Qilimanjaro's systems are truly coherent quantum annealers, implemented on a superconducting qubit quantum hardware platform. The use of superconducting flux qubits, combined with other key features such as advanced design for qubit-qubit interactions, is expected to yield available coherent quantum annealers in a competitively short time frame. The core focus of the Qilimanjaro technological efforts is to therefore maintain coherence in adiabatic quantum computations.

\footnotetext{
${ }^{12}$ Noisy Intermediate-Scale Quantum Technology, see https://quantum-journal.org/papers/q-2018-08-06-79/.

${ }^{13}$ https://www.nedo.go.jp/english/activities/activities_ZZJP_100123.html
} 


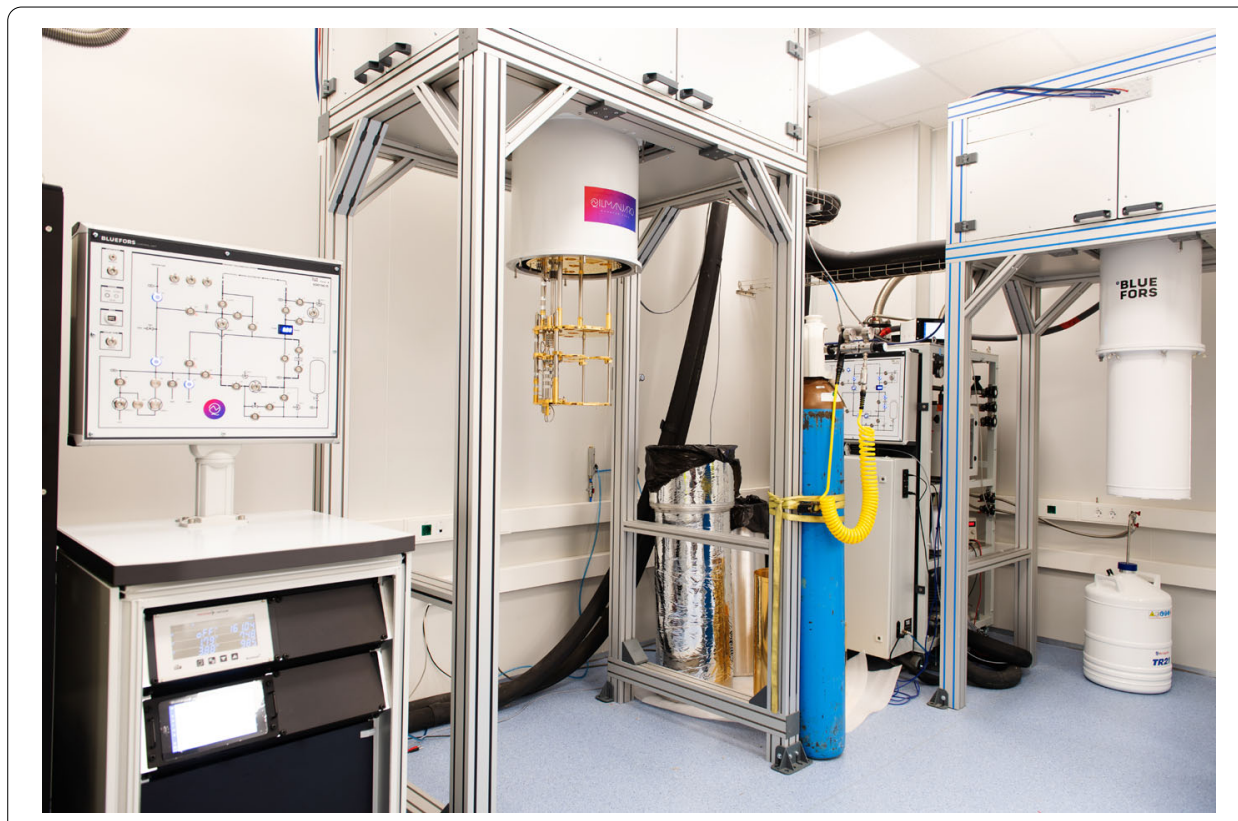

Figure 1 The Qilimanjaro lab, in its initial stages

Over the last decade, many experimental groups have contributed to prove the feasibility of implementing quantum computation using superconducting circuits: Coherence improvements were shown possible [17], several advanced control techniques have been developed [11, 18, 21], while both small- and medium-size algorithms have been implemented $[2,9]$. Prototypes already exist that contain these many building blocks, with full availability on the cloud. ${ }^{14,15}$ However, such building blocks are not only restricted to gatebased quantum computers. Qilimanjaro aims at leveraging these many improvements into its coherent quantum annealers, overcoming both coherence and circuit complexity problems of existing annealing platforms.

As outlined above, Qilimanjaro is a full partner of the FET-Open funded project $\mathrm{AVaQus}$. The project is coordinated by one of Qilimanjaro's founders at IFAE, with the aim of demonstrating a few-qubit prototype for coherent quantum annealing. This annealer prototype will contain full flexibility to implement various quantum annealing protocols, while at the same time representing a unit cell to be scaled up to a full-size quantum annealer (see Fig. 1).

The AVaQus project brings along several key ingredients mentioned before: (i) qubit coherence lasting the entire computation run-time, turning the system into a coherent quantum annealer; (ii) coupling designs and qubit connectivity layouts bringing enough complexity [14] to solve problems which are intractable for classical computers in larger-scale prototypes, allowing for the pursuit of quantum advantage; and iii) problem-tailored processor blueprints for specific problems with societal relevance, including quantum simulation instances in areas such as quantum chemistry [9].

The core architecture is thus compatible with the ideas of adiabatic universal quantum computation.

\footnotetext{
${ }^{14}$ https://quantum-computing.ibm.com/

${ }^{15}$ https://www.quantum-inspire.com/
} 


\section{QIBO: the software stack}

Quantum computation services are already offered remotely by a number of companies that deliver cloud services. While some of these services can be multiplied on cloud servers offering quantum simulations, there is a limited number of quantum devices connected to users. This connection requires a software service offering remote users the full functionality of quantum devices located in production laboratories, where specialized staff supervises and calibrates the correct operation of these quantum devices. A number of functionalities may appear on the wish list of users introduced into quantum computation, including execution control, a diversity of programming models, and tools easing access to a wide range of users with different scientific backgrounds. For these reasons, the Qilimanjaro platform aims to offer its users a set of powerful and easy-to-use programming tools to exploit its own devices.

Along the execution of algorithms on quantum hardware, users may take enormous advantage of the full simulation of quantum algorithms using classical methods. This approach offers insight beyond the limitations of quantum measurement, providing complete information of the transformation of quantum states and the result of a quantum algorithm.

However, simulations are limited by the exponential cost of representing a full quantum state, so many different approaches have been proposed to provide approximations to this representation, i.e. using different representations of the quantum state in terms of tensor networks that may overcome memory limitations by an algorithmic procedure to compute directly quantum observables. Notable examples of software simulating the exact representation of the quantum state are Cirq [16] and TensorFlow Quantum (TFQ) [5] from Google, Qiskit from IBM Q [15], PyQuil from Rigetti [19], Intel-QS (qHipster) from Intel [10], QCGPU [13] and Qulacs [20], among others.

Qilimanjaro develops, along with Abu Dhabi's Technology Innovation Institute, ${ }^{16}$ the open source Qibo platform [8] to offer remote services, including quantum computations on the coherent quantum annealer. This platform allows remote users to also choose among a collection of simulation backends, including simulation environments based on hardware acceleration (see the recent release of the Qibo code available at [7]). With these objectives in mind, Qibo is designed with the following target goals:

- a simple application programming interface (API) for quantum circuit design and adiabatic quantum computation.

- a high-performance simulation engine based on hardware acceleration tools.

- a clean design pattern to include classical/quantum hybrid algorithms.

The top layer of the Qibo stack (see Fig. 2) includes implementation of quantum algorithms, including variational quantum eigensolvers, time evolution of quantum systems, and error models among other applications. Further development by Qilimanjaro will extend this stack to offer quantum control capabilities using machine learning techniques, with an important impact to quantum optimization use cases. As a stack offering algorithmic services, Qibo will include implementation of quantum inspired algorithms so as to show the potential of classical tasks empowered by the quantum formalism.

${ }^{16} \mathrm{http}: / /$ www.quantum.tii.ae 


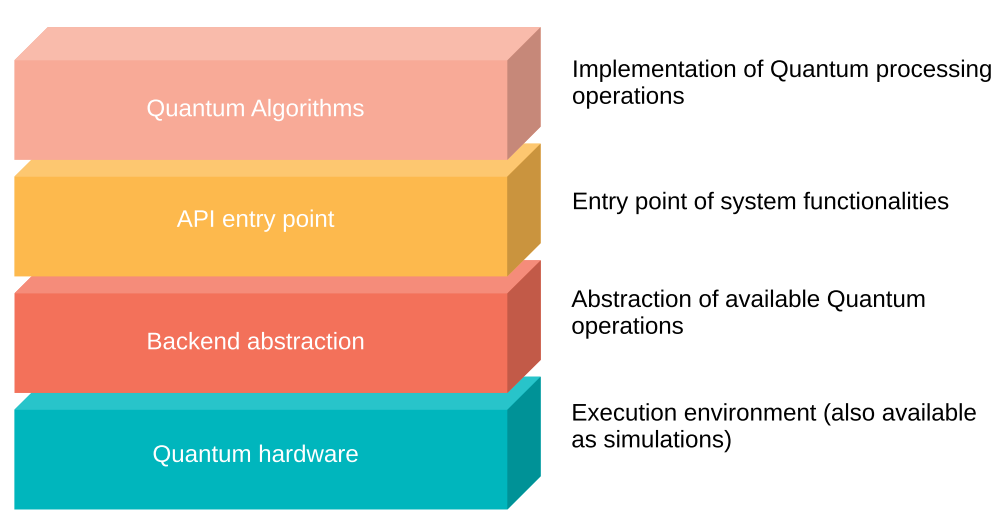

Figure 2 The Qibo stack structure

\section{Qilimanjaro's business model}

There are two clear phases in Qilimanjaro's business development, the initial one when the startup was created, and the one that will emerge as the company grows.

Qilimanjaro's qubit development requires access to specialized cryogenic and other laboratory infrastructure, such as clean rooms. Scaling of Qilimanjaro's coherent quantum annealer and algorithm design also requires access to advanced High Performance Computing (HPC) platforms for scaling up simulations. Both these requirements can be especially challenging to coordinate and costly to secure for a startup. This is where the multiple spin-off nature of Qilimanjaro became crucial.

The research group leaders of the quantum computing initiatives at the Institute for High Energy Physics (IFAE ${ }^{17}$ ) and at the Barcelona Supercomputing Center $\left(\mathrm{BSC}^{18}\right)$ are among Qilimanjaro's founders, both leading European research centers in experimental physics and HPC, respectively.

This fact paved the way to propose, negotiate and agree with these institutions the framework for cooperation, support and IP management. In addition, Qilimanjaro's third and senior scientific founder was a lead professor in quantum information science at the University of Barcelona $\left(\mathrm{UB}^{19}\right)$. The Qilimanjaro founders entered into a coordinated three-way negotiation process to agree on the conditions for becoming a spin-off of all three BSC, IFAE and UB.

The institutions were attracted to the idea of supporting a quantum initiative, and to contribute to further developing the quantum ecosystem in the Barcelona metropolitan area, including the QuantumCAT hub ${ }^{20}$ with the participation of the Institute of Photonics Sciences $\left(\mathrm{ICFO}^{21}\right)$, of whom one of the founders is a PhD alumni.

The three-way spin-off negotiation was successfully accomplished within a six-month period. A speedy resolution of this kind of spin-off agreements is really important for the successful launch of new quantum start-ups.

\footnotetext{
${ }^{17}$ www.ifae.es

${ }^{18}$ www.bsc.es

${ }^{19}$ www.ub.edu

${ }^{20}$ www.quantum-cat.cat

${ }^{21}$ www.icfo.eu
} 
As the founders were initially considering founding Qilimanjaro in 2018 and began communicating their plans, they were encouraged by the fact that the prestigious Boston Consulting Group captured their initiative in one of their executive white papers [4]. In addition, they were also approached by local business leaders in the financial and logistics industries, with the request to train them on, and to develop with them, innovative quantum algorithms, as well as quantum-inspired classical algorithms, that may prove critical to their business processes. Most of these use cases relate to different kinds of optimization and/or machine learning problems. These executives had become aware of the potential for quantum computing to eventually disrupt their businesses, so they were keen to become quantum ready.

Expertise in this field has been, and still is, limited to a group of individuals mostly in academic institutions. Reaching out to the academic experts was the natural thing to do, and this reflects a general opportunity for new spin-offs. This initially unsolicited demand for optimization and machine learning expertise, which happen to be key areas of applicability for adiabatic platforms, clinched the decision to found Qilimanjaro. Thus, the short-term opportunity is quantum algorithmic development, to be complemented later with access to an easy-to-use, powerful and affordable coherent quantum annealing platform. The algorithmic development is providing Qilimanjaro with initial client funding and, more importantly, provides it with true business use-case experiences, feeding back into the design objectives for the coherent quantum annealer platform.

Moreover, a similar request came up to provide quantum lab build-up consulting services and qubit development expertise. The combination of these initial client contracts has provided Qilimanjaro with an initial three-year funding runway-particularly having partnered with the research institutions of which it would become a multiple-way spin-off to leverage their own infrastructures.

Moving forward, Qilimanjaro's business objectives are twofold: to provide access to its coherent quantum annealing platform, and to offer its quantum algorithmic development expertise. Access for users to the platform will take place both via cloud services, as described above either direct and/or via established cloud services vendors, as well as via the acquisition of the platform itself. The latter scenario can be of interest for organizations willing to learn and further develop the qubit and/or the architecture itself, especially given one of Qilimanjaro's objectives of developing a physically small footprint system.

A key element of Qilimanjaro's future will be the establishment of strategic partnerships with other technology and/or IT organizations, so as to leverage their complementary offerings and as a go-to-market channel. Along these lines, Qilimanjaro will become one of the startups participating in the soon-to-be established European quantum industry consortium.

A note on the startup's name-why "Qilimanjaro"? The short answer is Q for quantum, and a combination of this $Q$ with the name of Africa's highest peak Kilimanjaro. Quantum computing is deemed to become a new IT paradigm, so relating Qilimanjaro to climbing the tallest free-standing peak was attractive-especially as one of the founders, an avid trekker, had enjoyed climbing it during his $\mathrm{PhD}$ times. So that's how the team came up with Qilimanjaro, the trail to lead users up to quantum computing readiness. 


\section{Concluding remarks}

In the coming years, Qilimanjaro shall strive to become a leading contributor to the European quantum computing scene, in terms of technology development and in terms of business success.

Its main goal is to significantly scale up the initial small-scale coherent quantum annealers with fast and efficient multiqubit readout and control, so as to act as a highly competitive general-purpose quantum simulator, an unparalleled optimizer for classical problems, and as a universal quantum computer in the longer run.

The unique circumstances related to Qilimanjaro's launch highlight some of the winning elements for successful quantum startups: a core team of enthusiastic, excellent scientists supported by professional business experts, that can access via spin-off agreements the required infrastructure resources (like clean rooms or HPC facilities) and initial funding (in Qilimanjaro's case, of the best kind, actual customer contracts). Funding is always key, and the typical sources are public grants or patient private venture capital funds. Qilimanjaro will be looking at all of them to further accelerate its growth.

The emerging quantum industry in Europe is ripe for many successful startups, given the wealth of its academic expertise and the growing awareness and interest from the market. All players in the ecosystem can play a key role in nurturing this development. Qilimanjaro is an example of a successful market launch, and the lessons learnt can be of interest for future spin-offs.

\section{Acknowledgements}

The authors acknowledge key contributions by all founders, as well as of the support of the three research institutions of which Qilimanjaro is a spin-off: Barcelona Supercomputing Center (BSC), Institute for High Energy Physics (IFAE) and University of Barcelona (UB)

\section{Funding}

Qilimanjaro is fully funded by client contracts. In addition it is a member of the H2020-FET-Open AVaQus grant agreement no. 899561 (November 2020).

Availability of data and materials

Does not apply.

Competing interests

The authors declare that they have no competing interests.

Authors' contributions

All authors have contributed to the write-up of their areas of responsibility: Dr. VC on the company strategy, Dr. PF-D and Mr. RS on the hardware architecture and Dr. AG-S on the software architecture. All authors read and approved the final manuscript.

\section{Authors' information}

Dr. Victor Canivell: Co-Founder, Chief Business Officer. Dr. Pol Forn-Díaz: Co-Founder, Chief Hardware Architect. Quantum Group leader at IFAE. Dr. Artur García Sáez: Co-Founder Chief Software Architect. Quantum Group leader at BSC. MSc. R. Sagastizabal: Senior Quantum Engineer.

\section{Author details}

'Qilimanjaro Quantum Tech S.L., Barcelona, Passeig de Gràcia 58, 08007 Barcelona, Spain. ${ }^{2}$ Institut de Física d'Altes Energies (IFAE), The Barcelona Institute of Science and Technology (BIST), Campus UAB, 08193 Bellaterra, Spain.

${ }^{3}$ Barcelona Supercomputing Center (BSC), C. Jordi Girona 29, 08034 Barcelona, Spain.

\section{Publisher's Note}

Springer Nature remains neutral with regard to jurisdictional claims in published maps and institutional affiliations.

Received: 7 January 2021 Accepted: 12 February 2021 Published online: 27 February 2021

References

1. Aharonov D, van Dam W, Kempe J, Landau Z, Lloyd S, Regev O. Adiabatic quantum computation is equivalent to standard quantum computation. 2007. https://doi.org/10.1137/S0097539705447323. 
2. Arute F, Arya K, Babbush R, Bacon D, Bardin JC, Barends R, Biswas R, Boixo S, Brandao FGSL, Buell DA, Burkett B, Chen Y, Chen Z, Chiaro B, Collins R, Courtney W, Dunsworth A, Farhi E, Foxen B, Fowler A, Gidney C, Giustina M, Graff R, Guerin K, Habegger S, Harrigan MP, Hartmann MJ, Ho A, Hoffmann M, Huang T, Humble TS, Isakov SV, Jeffrey E, Jiang Z, Kafri D, Kechedzhi K, Kelly J, Klimov PV, Knysh S, Korotkov A, Kostritsa F, Landhuis D, Lindmark M, Lucero E, Lyakh D, Mandrà S, McClean JR, McEwen M, Megrant A, Mi X, Michielsen K, Mohseni M, Mutus J, Naaman O, Neeley M, Neill C, Niu MY, Ostby E, Petukhov A, Platt JC, Quintana C, Rieffel EG, Roushan P, Rubin NC, Sank D, Satzinger KJ, Smelyanskiy V, Sung KJ, Trevithick MD, Vainsencher A, Villalonga B, White T, Yao ZJ, Yeh P, Zalcman A, Neven H, Martinis JM. Quantum supremacy using a programmable superconducting processor. 2019. http://www.nature.com/articles/s41586-019-1666-5.

3. Boothby K, Bunyk P, Raymond J, Roy A. White paper: Programming according to the fences and gates model for developing assured, secure software systems. Technical report, Next-Generation Topology of D-Wave Quantum Processors. D-Wave. 2019.

4. Boston Consulting Group. The next decade in quantum computing and how to play. https://www.bcg.com/publications/2018/next-decade-quantum-computing-how-play.

5. Broughton M, Verdon G, McCourt T, Martinez AJ, Yoo JH, Isakov SV, Massey P, Niu MY, Halavati R, Peters E, Leib M, Skolik A, Streif M, Von Dollen D, McClean JR, Boixo S, Bacon D, Ho AK, Neven H, Mohseni M. Tensorflow quantum: a software framework for quantum machine learning. 2020. https://arxiv.org/abs/2003.02989.

6. Brown KR, Kim J, Monroe C. Co-designing a scalable quantum computer with trapped atomic ions. 2016. https://doi.org/10.1038/npjqi.2016.34.

7. Efthymiou S, Carrazza S, bpcarlos, García-Martín D, Ramos S, Perez Salinas A Serrano J. Quantum-tii/qibo: Qibo 0.1.2. 2020. https://doi.org/10.5281/zenodo.4309870.

8. Efthymiou S, Ramos-Calderer S, Bravo-Prieto C, Pérez-Salinas A, García-Martín D, Garcia-Saez A, Latorre Jl, Carrazza S. Qibo: a framework for quantum simulation with hardware acceleration. 2020. https://arxiv.org/abs/2009.01845.

9. Google Al Quantum \& Collaborators, Arute F, Arya K, Babbush R, Bacon D, Bardin JC, Barends R, Boixo S, Broughton M, Buckley BB, Buell DA, Burkett B, Bushnell N, Chen Y, Chen Z, Chiaro B, Collins R, Courtney W, Demura S, Dunsworth A, Farhi E, Fowler A, Foxen B, Gidney C, Giustina M, Graff R, Habegger S, Harrigan MP, Ho A, Hong S, Huang T, Huggins WJ, loffe L, Isakov SV, Jeffrey E, Jiang Z, Jones C, Kafri D, Kechedzhi K, Kelly J, Kim S, Klimov PV, Korotkov A, Kostritsa F, Landhuis D, Laptev P, Lindmark M, Lucero E, Martin O, Martinis JM, McClean JR, McEwen M, Megrant A, Mi X, Mohseni M, Mruczkiewicz W, Mutus J, Naaman O, Neeley M, Neill C, Neven H, Niu MY, O'Brien TE, Ostby E, Petukhov A, Putterman H, Quintana C, Roushan P, Rubin NC, Sank D, Satzinger KJ, Smelyanskiy V, Strain D, Sung KJ, Szalay M, Takeshita TY, Vainsencher A, White T, Wiebe N, Yao ZJ, Yeh P, Zalcman A. Hartree-fock on a superconducting qubit quantum computer. 2020. https://science.sciencemag.org/content/369/6507/1084.

10. Guerreschi GG, Hogaboam J, Baruffa F, Sawaya NPD. Intel quantum simulator: a cloud-ready high-performance simulator of quantum circuits. 2020. https://doi.org/10.1088/2058-9565/ab8505.

11. Heinsoo J, Kraglund Andersen C, Remm A, Krinner S, Walter T, Salathé Y, Gasparinetti S, Besse J-C, Potočnik A, Wallraff A, Eichler C. Rapid high-fidelity multiplexed readout of superconducting qubits. 2018. https://link.aps.org/doi/10.1103/PhysRevApplied.10.034040.

12. IARPA. Quantum enhanced optimization (qeo). https://www.iarpa.gov/index.php/research-programs/qeo/qeo-baa

13. Kelly A. Simulating quantum computers using opencl. 2018. https://arxiv.org/abs/1805.00988.

14. Ozfidan I, Deng C, Smirnov AY, Lanting T, Harris R, Swenson L, Whittaker J, Altomare F, Babcock M, Baron C, Berkley AJ, Boothby K, Christiani H, Bunyk P, Enderud C, Evert B, Hager M, Hajda A, Hilton J, Huang S, Hoskinson E, Johnson MW, Jooya K, Ladizinsky E, Ladizinsky N, Li R, MacDonald A, Marsden D, Marsden G, Medina T, Molavi R, Neufeld R, Nissen M, Norouzpour M, Oh T, Pavlov I, Perminov I, Poulin-Lamarre G, Reis M, Prescott T, Rich C, Sato Y, Sterling G, Tsai N, Volkmann M, Wilkinson W, Yao J, Amin MH. Demonstration of a nonstoquastic hamiltonian in coupled superconducting flux qubits. 2020. https://link.aps.org/doi/10.1103/PhysRevApplied.13.034037.

15. Qiskit Team \& Collaborators. Qiskit: an open-source framework for quantum computing. 2019. https://qiskit.org/.

16. Quantum Al team and collaborators. Cirq. 2020. https://doi.org/10.5281/zenodo.4062499.

17. Reagor M, Pfaff W, Axline C, Heeres RW, Ofek N, Sliwa K, Holland E, Wang C, Blumoff J, Chou K, Hatridge MJ, Frunzio L, Devoret MH, Jiang L, Schoelkopf RJ. Quantum memory with millisecond coherence in circuit qed. 2016. https://ink.aps.org/doi/10.1103/PhysRevB.94.014506.

18. Rol MA, Battistel F, Malinowski FK, Bultink CC, Tarasinski BM, Vollmer R, Haider N, Muthusubramanian N, Bruno A, Terhal BM, DiCarlo L. Fast, high-fidelity conditional-phase gate exploiting leakage interference in weakly anharmonic superconducting qubits. 2019. https://link.aps.org/doi/10.1103/PhysRevLett.123.120502.

19. Smith RS, Curtis MJ, Zeng WJ. A practical quantum instruction set architecture. 2016. https://pyquil-docs.rigetti.com/en/stable/.

20. Suzuki Y, Kawase Y, Masumura Y, Hiraga Y, Nakadai M, Chen J, Nakanishi KM, Mitarai K, Imai R, Tamiya S, Yamamoto T, Yan T, Kawakubo T, Nakagawa YO, Ibe Y, Zhang Y, Yamashita H, Yoshimura H, Hayashi A, Fujii K. Qulacs: a fast and versatile quantum circuit simulator for research purpose. 2020. https://arxiv.org/abs/2011.13524.

21. Theis LS, Motzoi F, Machnes S, Wilhelm FK. Counteracting systems of diabaticities using drag controls: the status after 10 years. 2018. https://arxiv.org/abs/1809.04919. 